\title{
Quatro anos de experiência no tratamento trombolítico do AVC Isquêmico na cidade de Porto Alegre
}

\section{Four years of experience in thrombolytic therapy of ischemic stroke patients in Porto Alegre City}

Sheila Cristina Ouriques Martins ${ }^{1}$, Rosane Brondani', Maurício Friedrich², Mariana Dagnino Araújo3 , Adriano Wartchow ${ }^{3}$, Paulo Passos ${ }^{4}$, Euler Manenti ${ }^{5}$, Cristiano Jaeger ${ }^{6}$, Rafael Rech ${ }^{6}$, Daniel Silveira ${ }^{6}$, Karen Ruschel ${ }^{7}$, Luiz Antonio Nasi ${ }^{8}$, Márcia Lorena Fagundes Chaves $^{9}$, João Arthur Ehlers ${ }^{10}$

\section{RESUMO}

Embora o benefício do tratamento trombolítico no AVC isquêmico esteja bem demonstrado, o impacto na população geral de pacientes com AVC ainda é limitado, principalmente devido a estreita janela terapêutica. No Brasil, poucos centros estão estruturados para o uso do rtPA no AVC isquêmico agudo. Nós apresentamos os resultados de 4 anos de experiência em terapia trombolítica, com 173 pacientes tratados em 3 Unidades Vasculares implementadas em hospitais gerais no Sul do Brasil.

Unitermos: Acidente Cerebrovascular, Terapia trombolítica.

Citação: Martins SCO, Brondani R, Friedrich M, Araújo MD, Wartchow A, Passos P, Manenti E, Jaeger C, Rech R, Silveira D, Ruschel K, Nasi LA, Chaves MLF, Ehlers JA. Quatro anos de experiência no tratamento trombolítico do AVC Isquêmico na cidade de Porto Alegre. Rev Neurocienc 2006; 14(1):031-036.

\section{SUMMARY}

Although the benefit of thrombolytic treatment of stroke is well established, its impact in the general population with ischemic stroke is still limited primarily due to its narrow therapeutic window. In Brazil, few centers have the structure necessary for the use of rtPA in acute ischemic stroke patients. We present the results of the 4 years experience in thrombolytic therapy, with 173 patients treated in 3 Vascular Units established in general hospitals in south of Brazil.

\section{Keywords: Stroke, Thrombolytic therapy.}

Citation: Martins SCO, Brondani R, Friedrich M, Araújo MD, Wartchow A, Passos P, Manenti E, Jaeger C, Rech R, Silveira D, Ruschel K, Nasi LA, Chaves MLF, Ehlers JA. Four years of experience in thrombolytic therapy of ischemic stroke patients in Porto Alegre City. Rev Neurocienc 2006; 14(1):031-036.

Trabalho realizado: Hospital de Clínicas de Porto Alegre, Hospital Mãe de Deus e do Hospital São Lucas da Pontifícia Universidade Católica do Rio Grande do Sul. 1 - Neurologista vascular do Instituto de Medicina Vascular do Hospital Mãe de Deus (HMD) e da Unidade Vascular do Hospital de Clínicas de Porto Alegre (HCPA)

2 - Coordenador do programa de Doenças Neurovasculares do Hospital São Lucas da Pontifícia Universidade Católica do Rio Grande do Sul (HSL-PUCRS) e do Hospital Mãe de Deus (HMD)

3 - Neurologista Vascular do HSL-PUCRS e do Instituto de Medicina Vascular do HMD

4 - Neurorradiologista intervencionista do Instituto de Medicina Vascular do HMD

5 - Chefe da Unidade de Terapia Coronariana do HSL-PUCRS, responsável pela área de trombocardiologia do Instituto de Medicina Vascular do HMD

6 - Cardiologista do Instituto de Medicina Vascular do HMD

7 - Enfermeira de pesquisa do Instituto de Medicina Vascular do HMD

8 - Chefe do Serviço de Emergência e da Unidade Vascular do HCPA

9 - Chefe do Serviço de Neurologia do HCPA

10 - Coordenador Geral do Instituto de Medicina Vascular do Hospital Mãe de Deus

Endereço para correspondência: Sheila Cristina Ouriques Martins. Rua Engenheiro Olavo Nunes, 99/ 703. Bela Vista. CEP 90440-170. Porto Alegre-RS. 


\section{INTRODUÇÃO}

Com a aprovação do Ativador do Plasminogênio Tecidual recombinante (rtPA) em 1996 para utilização no tratamento do Acidente Vascular Cerebral Isquêmico (AVCl) com até 3 horas de evolução, a história natural desta doença começou a mudar ${ }^{1,2}$. Um dos fatores que possibilitou o emprego desta terapêutica foi a criação de uma estratégia de atendimento para o rápido reconhecimento, avaliação e tratamento do $\mathrm{AVCl}^{3}$.

Particularmente a abordagem do neurologista clínico evoluiu de uma atitude contemplativa para uma ação decisiva e de liderança no manejo destes pacientes. Tornou-se crítica a presença deste profissional capacitado para diagnosticar e tratar rapidamente todos os aspectos do AVC agudo 4 . Isto é mais evidente quando se considera a tomada de decisões para a indicação de novas opções terapêuticas intervencionistas como o tratamento intra-arterial, o tratamento combinado endovenoso e intraarterial, a trombectomia, a angioplastia de resgate e a hemicraniectomia ${ }^{5,6}$. Este novo perfil de atendimento do $\mathrm{AVCl}$, onde a reperfusão numa estreita janela terapêutica é o foco principal, fez surgir uma nova subespecialidade, a neurologia vascular. Além disso, foi necessária a formação de equipes multidisciplinares com treinamento específico nas diversas estratégias diagnósticas e terapêuticas do AVCl. Estas equipes multidisciplinares são formadas por neurologistas vasculares, neurorradiologistas, neurorradiologistas intervencionistas, neurocirurgiões e cardiointensivistas rapidamente acionados a partir da chegada do paciente ao hospital ${ }^{6}$.

No Brasil, o primeiro consenso para utilização de rtPA no AVCl foi publicado em 2001. Com o objetivo de utilizar a terapia de reperfusão no AVCl agudo, a partir de 2002 iniciamos a estruturação de centros para o atendimento destes pacientes na cidade de Porto Alegre. Atualmente, nossa equipe atua em 3 dos principais hospitais da cidade, Hospital Mãe de Deus (HMD), Hospital São Lucas da Pontifícia Universidade Católica do Rio Grande do Sul (HSL-PUCRS) e Hospital de Clínicas de Porto Alegre da Universidade Federal do Rio Grande do Sul (HCPA), com uma experiência acumulada de quase 200 pacientes tratados. Este artigo relata a experiência de 4 anos no tratamento trombolítico do $\mathrm{AVCl}$ agudo nestes 3 centros da cidade de Porto Alegre.

\section{MATERIAL E MÉTODO}

Todos os protocolos de atendimento foram submetidos aos comitês de ética dos 3 hospitais onde foi realizado este trabalho.

\section{Seleção de Pacientes}

Analisamos prospectivamente todos os pacientes com $\mathrm{AVCl}$ agudo tratados com terapia de reperfusão nos 3 hospitais de fevereiro de 2002 a abril de 2006 . Os critérios de inclusão e exclusão seguiram as recomendações da American Stroke Association (ASA) ${ }^{8,9}$.

\section{Estruturação dos centros}

Antes de iniciarmos o atendimento dos pacientes, todos os centros foram preparados com a criação das equipes de AVC disponíveis 24 horas por dia, implementação dos protocolos de trombólise e do fluxograma de atendimento, treinamento das equipes médica e de enfermagem, treinamento da aplicação da escala de AVC do National Institute of Health Stroke Scale $(\mathrm{NIHSS})^{10}$ e da escala de Rankin ${ }^{11}$ modificada, treinamento dos radiologistas para detecção de sinais precoces de isquemia na tomografia computadorizada (TC) de crânio, elaboração de termos de compromisso para a realização prioritária dos exames na radiologia e no laboratório, estabelecimento dos indicadores de qualidade no atendimento agudo do AVC e armazenamento prospectivo dos casos em banco de dados. Os indicadores de qualidade utilizados foram elegibilidade, taxa de sangramento cerebral sintomático, mortalidade, percentagem de pacientes com mínimo ou nenhum déficit neurológico em 3 meses (escore do NIHSS 0-1) e com mínima ou nenhuma incapacidade em 3 meses (Rankin modificado 0-1), tempo porta-tomografia e tempo porta-agulha (alvo de 45 e 60 minutos, respectivamente).

Todos os pacientes são acompanhados pela equipe de AVC desde o atendimento agudo até a alta hospitalar, sendo seguidos depois ambulatorialmente. Existem algumas particularidades na estrutura e na logística de atendimento de cada centro, descritas a seguir:

Hospital Mãe de Deus: Hospital geral, privado com 300 leitos, com uma Unidade Vascular (UV) localizada dentro da Emergência, inaugurada em dezembro de 2002 para atendimento agudo de pacientes com AVC, dor torácica e doença vascular periférica. Os pacientes são triados pela enfermeira da emergência, que identificam o possível caso de AVC e o transferem para a UV. Na UV o paciente é atendido pelo emergencista que aciona o protocolo de AVC, avisa o neurologista vascular de sobreaviso, solicita laboratório, TC crânio sem contraste e angiotomografia (angioTC) de crânio com contraste não iônico (para avaliar oclusão de grandes vasos). Na radiologia, o neurologista vascular e o neurorradiologista decidem juntos a indicação de tratamento de reperfusão e qual a melhor estratégia de tratamento para o caso. Se o tratamento for endovenoso (EV), o paciente retorna para a UV e inicia a infusão do rtPA. Se houver indicação de tratamento endovascular é acionado o neurorradiologista intervencionista (disponível 24 horas por dia) que geralmente em menos de 30 minutos está preparado na sala de hemodinâmica (também disponível 24 horas por dia, 7 dias por sema- 
na). Após o tratamento, o paciente permanece na UV ou é transferido para a Unidade de Tratamento Intensivo. Do início do tratamento até a alta hospitalar o paciente é sempre acompanhado por um cardiointensivista da equipe de AVC.

Hospital São Lucas da PUC: Hospital geral, privado, universitário com 600 leitos, 50\% deles destinados ao SUS. A Unidade Vascular, localizada na Unidade de Terapia Coronariana (UTC), iniciou seu funcionamento em fevereiro de 2002. Os pacientes são triados pelo médico emergencista e quando identificado um caso de AVC, transferidos para a sala de Urgência, onde são monitorizados, coletado sangue, solicitada TC de crânio sem contraste e acionado o residente da neurologia. Este avalia o paciente, aciona o neurologista vascular de sobreaviso e leva o paciente para a radiologia. Após a TC, se for indicada a trombólise, o paciente é transferido para a UV, onde é tratado. Se houver indicação de terapia endovascular aguda, é acionado o radiologista intervencionista e, se a hemodinâmica estiver disponível, o paciente recebe tratamento intra-arterial.

Hospital de Clínicas de Porto Alegre: Hospital geral e universitário, com 749 leitos, com um Serviço de Emergência que atende aproximadamente 6000 pacientes do SUS por mês. Após a reestruturação física da Emergência foi criada a Unidade Vascular, que passou a funcionar em de 28 de dezembro de 2005, com 5 leitos exclusivos para o atendimento do paciente com doença vascular aguda: AVC, síndrome coronariana aguda, doenças agudas da aorta e tromboembolismo pulmonar. Desde agosto de 2005 a Emergência adotou uma nova sistemática de atendimento baseada em critérios de risco para todos os pacientes que procuram o hospital. Os pacientes são triados pelo enfermeiro e, se houver suspeita de doença vascular, são classificados como de alto risco. Convencionou-se que estes pacientes são identificados pela cor vermelha e que, portanto, recebem atendimento priorizado. Dentro de 10 minutos da chegada, realizam eletrocardiograma e são encaminhados para a Unidade Vascular. Imediatamente o médico residente ou o emergencista de plantão avalia o paciente e desencadeia o protocolo de AVC: aciona o neurologista vascular, solicita laboratório e TC de crânio sem contraste. Após a coleta de sangue, o paciente é levado à radiologia, acompanhado pelo residente. $\mathrm{O}$ neurologista vascular, após avaliação do paciente, da TC e dos exames de sangue, inicia a trombólise EV na UV. O paciente pós trombólise estável permanece de 36 a 72 horas monitorizado na UV, sendo depois transferido para um leito de enfermaria.

\section{Protocolo de Tratamento}

O protocolo de tratamento com rtPA EV para o AVCI dentro de 3 horas do início dos sintomas é o mesmo em todos os centros e segue as recomendações da
American Stroke Association ${ }^{8,9}$. É administrada a dose de $0,9 \mathrm{mg} / \mathrm{kg}$ de rtPA, com $10 \%$ em bolus em 1 minuto e o restante em infusão de 1 hora (no máximo 90 mg).

À partir de 2004, com a publicação da análise combinada dos ensaios clínicos ATLANTIS, ECASS, e $\mathrm{NINDS}^{12}$, que mostrou benefício do tratamento além da janela de 3 horas, começamos a utilizar tratamento EV também para alguns pacientes selecionados com início dos sintomas entre 3 e 5 horas.

No HMD, os pacientes com oclusão de T carotídeo e oclusão da artéria basilar são submetidos à tratamento intra-arterial: trombólise mecânica + rtPA até a dose máxima de $30 \mathrm{mg}$. Se este tratamento não for efetivo, é realizada angioplastia do vaso ocluído. Os pacientes com oclusão proximal da artéria cerebral média dentro de 3 horas do início dos sintomas recebem tratamento endovenoso e, se não houver melhora do déficit durante a infusão, são levados para arteriografia. Se ainda houver oclusão do vaso, recebem tratamento de resgate intra-arterial (trombólise mecânica e, se necessário, angioplastia). Os pacientes com oclusão proximal da artéria cerebral média com mais de 3 horas de evolução são submetidos à tratamento intra-arterial.

NO HSL-PUCRS, os pacientes com oclusão de carótida ou oclusão de artéria basilar entre 0 e 6 horas recebem tratamento intra-arterial.

\section{RESULTADOS}

Foram analisados 173 pacientes com AVC isquêmico, tratados com terapia de reperfusão, nos 3 hospitais no período de fevereiro de 2002 a abril de 2006. No HMD foram tratados 85 pacientes (60 EV e $25 \mathrm{IA}$ ) no período de dezembro de 2002 a abril de 2006, no HSL-PUCRS 76 (70 EV e 6 IA) no período de fevereiro de 2002 a novembro de 2005 e no HCPA 12 (todos EV) no período de janeiro a abril de 2006. A taxa de elegibilidade para o tratamento foi de $14 \%$.

A média de idade foi de 67 anos (24-94), 69\% mulheres e $92 \%$ brancos. O fator de risco mais freqüente foi hipertensão (77\%) e o subtipo de AVC mais comum foi cardioembólico (54\%) seguido por aterotrombótico de grandes vasos (37\%). O tempo médio do início dos sintomas até a chegada na emergência foi de 92 minutos.

Nos 142 pacientes, que receberam tratamento endovenoso, o tempo do início dos sintomas até o tratamento com rtPA foi de 159 minutos. O tempo porta-tomografia foi de 26 minutos e o tempo porta-agulha de 72 minutos. Cinqüenta e dois porcento dos pacientes (74) receberam trombolítico dentro dos primeiros 60 minutos da chegada ao hospital.

O escore médio do NIHSS na chegada foi de $12( \pm$ $6,7)$, na alta de 3,8 $( \pm 5,3)$ e em 3 meses $2,6( \pm 3,5)$. Cinqüenta e quatro porcento dos pacientes apresen- 
taram-se sem déficit neurológico (NIHSS 0-1) ou incapacidade funcional (Rankin modificado 0-1) ao final de 3 meses. Independência funcional (Rankin modificado 0-2 em 3 meses) foi obtida em $64 \%$ dos pacientes. A evolução dos pacientes em 3 meses de acordo com o escore inicial do NIHSS é demonstrada na figura 1. Dos pacientes que chegaram ao hospital com escore NIHSS baixo (entre 0 e 5), 90\% ficou sem déficit neurológico em 3 meses enquanto daqueles que chegaram com NIHSS alto (acima de 20), apenas 20\% ficou sem déficit.

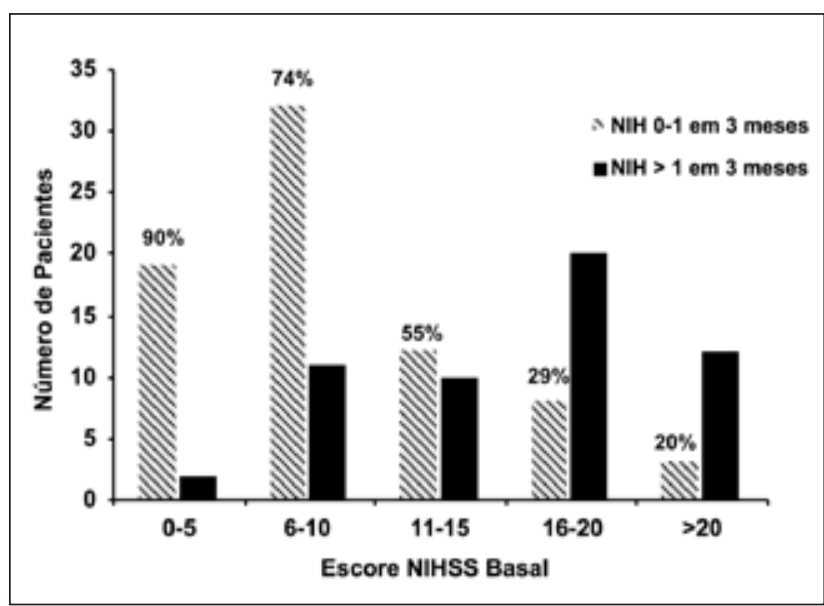

Figura 1. Evolução dos pacientes após 3 meses da trombólise endovenosa de acordo com o escore inicial do National Institute of Health Stroke Scale - NIHSS ( $n=142)$. Escore NIHSS entre 0 e 1 foi considerado boa evolução. O percentual destacado acima das colunas listradas indica a proporção de pacientes que obtiveram boa evolução dentro de cada intervalo do NIHSS basal.

Quatro pacientes (2,8\%) apresentaram reação alérgica ao rtPA. Angioedema ocorreu em 3 pacientes (2 deles com comprometimento de glote) e choque refratário em 1. Hemorragia intracraniana sintomática ocorreu em 9 pacientes (6,3\%). A mortalidade foi de 15,5\%, com apenas $27 \%$ dos óbitos sendo relacionados ao rtPA (5 pacientes devido a sangramento cerebral e 1 paciente devido a anafilaxia com choque refratário). Demais óbitos foram secundários a infarto agudo do miocárdio, infarto cerebral extenso em território carotídeo ou artéria cerebral média e complicações infecciosas.
A tabela 1 apresenta os nossos resultados comparados com os estudos NINDS, CASES (registro canadense de trombólise em 60 centros) ${ }^{13}$ e com outras coortes de trombólise com rtPA EV ${ }^{5,14}$.

A tabela 2 demonstra os resultados dos pacientes que receberam tratamento endovascular nos Hospitais Mãe de Deus e Hospital São Lucas. Dezessete pacientes foram submetidos a trombólise intra-arterial com rtPA + lise mecânica, 3 foram submetidos à trombectomia (oclusão de artéria basilar com mais de 6 horas de evolução apresentando mismatch na RM com difusãoperfusão), 2 foram tratados com angioplastia seguida de colocação de stent (1 devido a estenose $>50 \%$ no tronco da artéria cerebral média e 1 devido à extensa dissecção da carótida interna), 1 recebeu exclusivamente lise mecânica (oclusão de ACM sem definição de tempo do início dos sintomas mas com presença de grande área de mismatch) e 8 pacientes receberam rtPA endovenoso seguido de resgate intra-arterial (oclusão de tronco da artéria cerebral média). A figura 2 apresenta os escores do NIHSS na chegada ao hospital e 3 meses após o tratamento.

\section{DISCUSSÃo}

Através da organização de centros para atendimento do AVCl agudo criou-se condições para o emprego seguro da terapia trombolítica, tanto endovenosa quanto intra-arterial. Com esta estratégia de atendimento foi possível tratar $14 \%$ dos pacientes com AVCl, obtendo-se taxas de hemorragia sintomática com o uso do rtPA endovenoso semelhantes às reportadas na literatura ${ }^{1,5,6}$.

Nossa taxa de elegibilidade é maior que a maioria dos hospitais norte americanos (geralmente menor que $5 \%$ ) e similar a centros internacionais estruturados para atendimento do $\mathrm{AVC}^{5}$.

Os tempos porta-tomografia de 26 minutos e porta-agulha de 72 minutos demonstram que um centro preparado para o atendimento permite uma rápida investigação e tratamento. O tempo porta-agulha menor que 60 minutos, recomendado como alvo para os

Tabela 1. Comparação dos resultados da trombólise endovenosa (EV) em Porto Alegre com os resultados dos estudos NINDS, CASES e coortes de outros centros.

\begin{tabular}{lccccc}
\hline $\mathbf{n}$ & $\begin{array}{c}\text { NINDS } \\
\mathbf{( 3 1 2 )}\end{array}$ & $\begin{array}{c}\text { CASES } \\
\mathbf{( 1 1 3 5 )}\end{array}$ & $\begin{array}{c}\text { Cologne } \\
\mathbf{( 1 0 0 )}\end{array}$ & $\begin{array}{c}\text { Houston } \\
\mathbf{( 2 6 9 )}\end{array}$ & $\begin{array}{c}\text { Porto Alegre } \\
\mathbf{( 1 4 2 )}\end{array}$ \\
\hline Elegibilidade (\%) & 4,2 & - & 22 & 15 & 14 \\
Tempo porta-agulha (min.) & - & 85 & 48 & 70 & 72 \\
NIHSS 0/1 3 meses (\%) & 31 & 25 & 53 & - & 54 \\
Mortalidade (\%) & 17,6 & 22 & 20 & 15 & 15,5 \\
Hemorragia cerebral sintomática (\%) & 6,4 & 4,6 & 5 & 4.5 & 6,3 \\
\hline
\end{tabular}

NIHSS $=$ National Institute of Health Stroke Scale. 
Tabela 2. Características dos pacientes e resultados do tratamento intra-arterial

\begin{tabular}{ll}
\hline Número de pacientes & $\mathbf{3 1}$ \\
\hline NIHSS inicial & $22(4-40)$ \\
Pacientes com NIHSS inicial > 15 & $20(65 \%)$ \\
NIHSS em 3 meses & $5,3(0-12)$ \\
NIHSS 0-1 em 3 meses & $31 \%$ \\
Independência Funcional & $41 \%$ \\
Hemorragia Cerebral Sintomática & $25 \%$ \\
Óbito (n) & $10(32 \%)$ \\
Óbitos por sangramento (n) & $5(16 \%)$ \\
Tempo porta-agulha & 95 minutos \\
\hline
\end{tabular}

NIHSS $=$ National Institute of Health Stroke Scale.

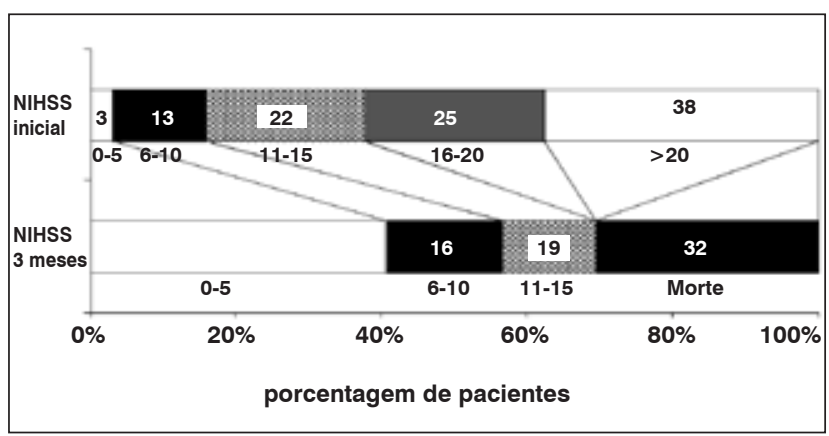

Figura 2. Escores do National Institute of Health Stroke Scale - NIHSS na admissão hospitalar (NIHSS inicial) e em 3 meses nos pacientes com tratamento de reperfusão intra-arterial.

centros que tratam $\mathrm{AVCl}$ agudo ${ }^{3,8}$, é considerado um indicador de qualidade do atendimento. Quanto maior a proporção de pacientes tratados dentro deste limite, melhor está organizado o centro. Na prática, entretanto, na maioria dos hospitais apenas 14 a 20\% dos pacientes atingem esta meta ${ }^{5}$. Em nossas UV, 52\% dos pacientes receberam rtPA em menos de 60 minutos da chegada. O fato de o neurologista estar, na maior parte do tempo, dentro do hospital pode ter contribuído para a agilidade do tratamento.

Cinqüenta e quatro por cento dos pacientes apresentaram mínimo ou nenhum déficit em 3 meses $(\mathrm{NIH}$ $0-1)$. Este percentual é semelhante ou um pouco superior ao encontrado em outras séries internacionais (tabela 1).

Pacientes muito graves (escore médio do $\mathrm{NIH}$ da chegada de 22, com $63 \%$ dos pacientes com $\mathrm{NIH}>15$ ) foram submetidos a tratamento intra-arterial. Destes, $31 \%$ apresentaram-se sem déficits em 3 meses e 41\% tinham independência funcional. Apesar da taxa de sangramento de $25 \%$ ser maior que as descritas no tratamento intra-arterial ${ }^{15-17}$ (aproximadamente 10-15\%), ela é compatível com a gravidade dos casos tratados. Além disso, a taxa mortalidade de $31 \%$ é até menor que a esperada nesta situação. Este é um excelente resultado considerando-se um grupo de pacientes cuja evolução natural geralmente é catastrófica, com a maioria deles evoluindo para o óbito ou alto grau de incapacidade (principalmente no caso dos 4 pacientes com oclusão da artéria basilar, 3 deles com mais de 6 horas de evolução, e dos 7 pacientes com oclusão de T carotídeo). Em uma análise de subgrupos do estudo NINDS ${ }^{18}, 52 \%$ dos pacientes tratados com rtPA endovenoso com NIHSS $<10$ evoluíram para NIHSS 0 ou 1, enquanto somente $8 \%$ dos pacientes com NIHSS $>20$ tiveram boa evolução. A explicação para a pior evolução nos pacientes com déficit neurológico moderado a grave é que, geralmente, estes pacientes têm oclusões de grandes artérias que, na maioria das vezes, não recanaliza com rtPA endovenoso no tempo necessário para previnir o infarto cerebral ${ }^{19}$. As taxas de recanalização parcial ou completa com tratamento EV de oclusões da artéria carótida interna são de 10\% e das oclusões proximais da artéria cerebral média $25 \%{ }^{20,21}$. Recente metanálise ${ }^{17}$ avaliou 27 estudos de tratamento intra-arterial de oclusão proximal da artéria cerebral média e sistema vertebrobasilar, num total de 852 pacientes e 100 controles. Em 90 dias 41,5\% dos tratados tiveram boa evolução x $23 \%$ dos controles, com taxa de recanalização de $72 \%$. A taxa de hemorragia intracraniana foi de $9,5 \%$ e a mortalidade de $27,2 \%$.

Acreditamos que o manejo por uma equipe de AVC multidisciplinar melhora a evolução dos pacientes ${ }^{6}$, desde a decisão da melhor estratégia terapêutica, muitas vezes tomada em conjunto pelo neurologista vascular, neurorradiologista, radiologista intervencionista e cardiointensivista, passando pelo controle rígido de pressão arterial durante e após a trombólise, até o manejo das complicações cardiovasculares, freqüentemente concomitantes ao evento agudo.

Enfatizamos a importância do treinamento continuado para garantir a manutenção do sistema de atendimento, além da monitorização dos indicadores de qualidade para avaliar possíveis problemas, possibilitando a reestruturação das estratégias e das metas.

Finalmente, demonstramos que é possível implementar terapia trombolítica em hospitais com diferentes estruturas, tendo como base um local adequado para o tratamento, uma equipe de AVC, uma estrutura organizada e treinamento adequado e contínuo. Demonstramos também que locais com alta demanda de pacientes do SUS, se bem organizados, também podem oferecer tratamento trombolítico com os mesmos resultados. 


\section{REFERÊNCIAS BIBLIOGRÁFICAS}

1. The National Institute of Neurological Disorders and Stroke rtPA Stroke Study Group (NINDS). Tissue plasminogen activator for acute ischemic stroke. N Engl J Med 1995;333:1581-1587

2. Adams HP, Brott TG, Furlan AJ, Gomez CR, Grotta J, Helgason CM, et al. Guidelines for thrombolytic therapy for acute stroke: a supplement to the guidelines for the management of patients with acute ischemic stroke. Circulation 1996;94:1167-1174

3. The National Institute of Neurological Disorders and Stroke rt-PA Stroke Study Group (NINDS). A systems approach to immediate evaluation and management of hyperacute stroke: experience at eight centers and implications for community practice and patient care. Stroke 1997;28:1530-1540.

4. Schwamm LH, Pancioli A, Acker III JE, Goldstein LB, Zorowitz RD, Shepard TJ, et al. Recommendations from the American Stroke Association's Task Force on the development of stroke systems. Stroke 2005; 36: 690-703.

5. Grotta JC, Burgin WS, Mitwalli A, Long M, Campbell M, Morgenstern LB et al. Intravenous tissue-type plasminogen activator therapy for ischemic stroke: Houston experience 1996-2000. Arch Neurol 2001;58:2009-2013.

6. Katzan IL, Hammer MD, Furlan AJ, Hixson ED, Nadzan DM. Cleveland Clinic health system stroke quality improvement team. Quality improvement and tissue-type plasminogen activator for acute ischemic stroke: a Cleveland update. Stroke 2003;34: 799-800.

7. Sociedade Brasileira de Doenças Cerebrovasculares. Primeiro consenso brasileiro do tratamento da fase aguda do acidente vascular cerebral. Arq Neuropsiquiatr 2001;59:972-980

8. Adams HP, Adams RJ, Brott T, del Zoppo GJ, Furlan A, Goldstein LB, et al Stroke Council of the American Stroke Association. Guidelines for the early management of patients with ischemic stroke: a scientific statement from the stroke council of the American Stroke Association. Stroke 2003;34:1056-83.

9. Adams H, Adams R, Del Zoppo G, Goldstein LB. Guidelines for the early management of patients with ischemic stroke: a scientific statement from the stroke council of the American Stroke Association. Stroke 2005;36:916-921.

10. Brott T, Adams HP, Olinger CP, Marler JR, Barsan WG, Biller J, et al. Measurements of acute cerebral infarction: a clinical examination scale. Stroke $1989 ; 20: 864-870$
11. Rankin J. Cerebral vascular accidents in patients over the age of 60: II. Prognosis. Scot Med J 1957; 2: 200-215

12. The ATLANTIS, ECASS, and NINDS rtPA Study Group Investigators. Association of outcome with early stroke treatment: pooled analysis of ATLANTIS, ECASS, and NINDS rtPA stroke trials. Lancet 2004;363:768-774.

13. Hill MD, Buchan AM, for the Canadian Alteplase for Stroke Effectiveness Study (CASES) Investigators. Thrombolysis for acute ischemic stroke: results of the Canadian Alteplase for Stroke Effectiveness Study. CMAJ 2005:172:1307-1312.

14. Grond M, Stenzel C, Schmulling S, Rudolf J, Neveling M, Lechleuthner A, et al. Early intravenous thrombolysis for acute ischemic stroke in a community-based approach. Stroke 1998;29:1544-1549.

15. Furlan A, Higashida R, Wechsler L, Gent M, Rowley H, Kase C, et al. Intraarterial prourokinase for acute ischemic stroke: the PROACT II study: a randomized controlled trial. JAMA 1999;282:2003-2011.

16. del Zoppo GJ, Higashida RT, Furlan AJ, Pessin MS, Rowley HA, Gent M PROACT: A Phase II Randomized Trial of Recombinant Pro-Urokinase by Direct Arterial Delivery in Acute Middle Cerebral Artery Stroke. Stroke 1998;29:4-11.

17. Lisboa RC, Jovanovic BD, Alberts MJ. Analysis of the safety and efficacy of intraarterial thrombolytic therapy in ischemic stroke. Stroke 2002;33:2866-2871.

18. The National Institute of Neurological Disorders and Stroke rt-PA Stroke Study Group (NINDS). Generalized efficacy of t-PA for acute stroke: subgroup analysis of the NINDS t-PA stroke trial. Stroke 1997;28:2119-2125.

19. Ernst R, Pancioli A, Tomsick T, Kissela B, Woo D, Kanter D, et al. Combined intravenous and intra-arterial recombinant tissue plasminogen activator in acute ischemic stroke. Stroke 2000;31:2552-2557.

20. Wolpert S, Bruckmann H, Greenlee R, Wechsler L, Pessin M, del Zoppo G Neuroradiologic evaluation of patients with acute stroke treated with recombinant tissue plasminogen activator. Am J Neuro Radiol 1993;14:3-13.

21. Mori E, Yoneda Y, Tabuchi M, Yochida T, Ohkawa S, Ohsumi Y, et al. Intravenous recombinant tissue plasminogen activator in acute carotid artery territory stroke. Neurology 1992;1992:976-982.

\section{ABRASPI}

\section{Associação Brasileira da Síndrome das Pernas Inquietas}

A Associação Brasileira da Síndrome das Pernas Inquietas - ABRASPI - É uma Instituição sem fins lucrativos criada para dar suporte a pacientes e promover pesquisa e treinamento sobre a Síndrome das Pernas Inquietas (SPI). Criada desde 2005, a ABRASPI tem recebido e-mails de pacientes, familiares e médicos do Brasil todo e de Portugal, que procuram tirar dúvidas sobre a doença, tratamento e indicações de médicos. Nesse período, foram 268 consultas com encaminhamentos, 30 médicos cadastrados, 100 associados e 3167 visitas ao site

Rua Claudio Rossi, 394, São Paulo - SP, CEP 01547-000. Fone/fax 551150816629.

sindromedaspernasinquietas@gmail.com, sindromedaspernas@hotmail.com

www.sindromedaspernasinquietas.com.br 